\title{
Image Retrieval Based on Fuzzy Mapping of Image Database and Fuzzy Similarity Distance
}

\author{
Siddhivinayak Kulkarni \\ School of Information Technology and Mathematical Sciences \\ University of Ballarat, P. O. BOX 663, Ballarat, Victoria, 3353, Australia \\ E-mail: S.Kulkarni@ballarat.edu.au
}

\begin{abstract}
The on-line image retrieval process consists of a query example image, given by the user as an input, from which low-level image features are extracted. These image features are used to find images in the database which are most similar to the query image. A drawback, however, is that these low level image features are often too restricted to describe images on a conceptual or semantic level. The gap between the high level query from the user and low level features extracted by a computer is known as the semantic gap. Translating or converting the question posed by a human to the low level features seen by the computer illustrates the problem in bridging the semantic gap. This paper proposes a novel fuzzy approach for mapping the fuzzy database while extracting the colour features from image and assigning the weights to this fuzzy content when calculating the similarity between the query image and the images in database. Number of experiments was conducted on a small colour image database and promising results were obtained.
\end{abstract}

\section{Introduction}

The size of the digital image collection is increasing very rapidly due to the advancement in technological devices. These images are stored digitally and transmitted over the Internet at a very high speed. To retrieve the images based on their content effectively and efficiently further processing of the images is essential. But how to retrieve the images based on their content? There are few image retrieval systems developed commercially as well as academically.

Most of the Content-based Image Retrieval (CBIR) systems such as QBIC [1], Virage [2], Photobook [3] and Netra [4] use a weighted linear method to combine similarity measurements of different feature classes. QBIC [1] executes the queries by calculating the similarity between the pre-extracted features of the images in a database. QBIC allows queries based on example images, user-constructed sketches or/and selected colour and texture patterns. The percentage of a specific colour in an image is adjusted by moving sliders. To perform a query in Photobook [3], the user selects some images from the grid of still images displayed and/or enters annotation filter. The images obtained with the query are refined to make another search. VisualSEEK [5] determines the similarity by measuring image regions using both colour parameters and spatial relationships. To pose a query, user sketches a number of positions and dimensions them on the grid and selects a colour for each region. Also, the user can indicate boundaries for location and size and spatial relationships between regions. Netra [4] depends upon image segmentation to carry out region based searches that allow the user to select example regions and lay emphasis on image attributes to focus the search. The user can select any one image as query image from 2500 images, clustered into 25 classes and 100 images for each class. The images are segmented into various homogenous regions and the user can select any one of the region for possible matching based on colour, texture, spatial location and shape. The main motivation for the development of this system is that region-based search improves the quality of the image retrieval. Therefore the system incorporates an automated region identification algorithm. Region-based querying is also used in Blobworld [6] where global histograms are shown to perform comparatively poorly on images containing distinctive objects. The user first selects a category, which already limits the search space. In an initial image, the user selects a region (blob), and indicates the importance of the blob. Next, the user indicates the importance of each blob based on fuzzy terms such as 'not', 'somewhat', 'very'. Multiple regions are used for querying. In Chabot [7], the user is presented with a list of search criteria such as keywords, colours, photographer etc. The colour criterion offers the options in terms of specific content of the each colour. The concept in an image is demonstrated by a 
descriptive keyword and specific colour related to the keyword.

Fuzzy logic offers a good solution for posing a query in terms of natural language based on the various features of an image [8]. Fuzzy logic has been extensively used at various stages of image retrieval such as region groupings within the images as a feature extraction technique, fuzzy image segmentation, recognition of fuzzy objects etc. Fuzzy logic is proposed for the computation of fuzzy colour histogram as well as posing the queries in CBIR. Han and $\mathrm{Ma}$ [9] propose a fuzzy colour histogram that permits to consider colour similarity across different bins and the colour dissimilarity between the different bins. Colour naming system proposed by Sugano [10] describes a technique to convert colour into set of words such as dark red by using fuzzy membership function to define saturation and lightness for a given hue. The problem of image indexing and retrieval of colour images is demonstrated in [11]. The lightness and saturation are represented through linguistic qualifiers also defined in fuzzy way. This paper proposes a novel technique based on fuzzy logic to reduce the semantic gap. Colour image features are mapped to form image feature database and weights are used to calculate the similarity based on fuzzy logic between the two images. The rest of the paper is organised as follows: Section 2 proposes fuzzy mapping of image feature database, Section 3 details the similarity function based on fuzzy logic, experimental results for colour image retrieval are discussed in Section 4, these results are compared and analysed in Section 5 and the paper is concluded in Section 6.

\section{Fuzzy mapping}

Colour feature extraction forms the basis of colour image retrieval. The distribution of colour is a useful feature for image representation. Colour distribution, which is best represented as a histogram of intensity values, is more appropriate as a global property which does not require knowledge of how an image is composed of different objects. So this technique works extremely well to extract global colour components from the images. A colour histogram technique is used for extracting the colours from the images. The colour of any pixel may be represented in terms of the components of red, green and blue values. These histograms are invariant under translation and rotation about the view axis and change only under the change of angle of view, change in scale and occlusion. Therefore, the colour histogram is a suitable quantitative representation of image content.
Let $F_{S}$ denote the set of features used to represent colour content, $\mathrm{F}_{\mathrm{S}}=$ \{colour $\}$. The feature representation set of colours is rep (colour) $=$ \{red, green, blue, white, black, yellow, orange, pink, purple\}.

An image histogram refers to the probability mass function of the image intensities. This is extended for colour images to capture the joint probabilities of the intensities of the three-colour channels. More formally, the colour histogram is defined by

$$
h_{r g b}=N \cdot \operatorname{prob}\{R=r, G=g, B=b\} \text { wher }
$$

e R, G, B represent the three-colour channels and $\mathrm{N}$ is the number of pixels in an image. These RGB values are converted into Hue $[0,360]$, Saturation $[0,1]$ and Value $[0,1]$.

Maximum and minimum values for RGB were calculated. Saturation (S) is the ratio of the difference between maximum and minimum values to the maximum value [12]. After getting these terms, HSV were calculated.

Algorithm RGB_to_HSV (r, g, b:real; var h, s, v:real)

\{Given: $r, g, b$, each in $[0,1]$.

Desired: $\mathrm{h}$ in $[0,360), \mathrm{s}$ and $\mathrm{v}$ in $[0,1]$ expect if $\mathrm{s}=0$, then $\mathrm{h}=\mathrm{UNDEFINED}$

begin

Red $->$ val[RED] > val[GREEN] + val[BLUE]

Green $\rightarrow>\operatorname{val}[$ GREEN] > val[BLUE] \&\& val[Green] > $\operatorname{val}[\mathrm{RED}]$

BLUE -> val[BLUE] $>0.5$ (val[RED] + val[GREEN])

WHITE $->\operatorname{val}[$ RED] $>200 \& \& \operatorname{val}[$ BLUE] $>200 \& \&$ $\operatorname{val}[$ GREEN] $>200$

BLACK -> val[RED] $<30 \& \&$ val[GREEN] $<30 \& \&$ $\operatorname{val}[$ BLUE] $<30$

YELLOW $->\mathrm{h}>42 \& \& \mathrm{~h}<62 \& \& \mathrm{~s}>0.6 \& \& \mathrm{v}>$ 0.95

ORANGE $->\mathrm{h}>2 \& \& \mathrm{~h}<40 \& \& \mathrm{~s}>.7 \& \& \mathrm{v}>.93$

PINK- $>\mathrm{h}>320 \& \& \mathrm{~s}<.5$

PURPLE $->(\mathrm{h}>320 \& \& \mathrm{~h}<330) \& \&(\mathrm{~s}>.50) \& \&$ $(\mathrm{v}>.60 \& \& \mathrm{v}<.8)$

end

The fuzzy contents of the image are expressed as follows:

$\mathrm{F}_{\text {contents }}=$ \{verylarge, large, ratherlarge, medium, rathersmall, small, verysmall\}

Instead of having numerical feature values, the values are mapped based on fuzzy logic and stored in feature database. Figure 2 describes the steps of storing the fuzzy content of an image. 


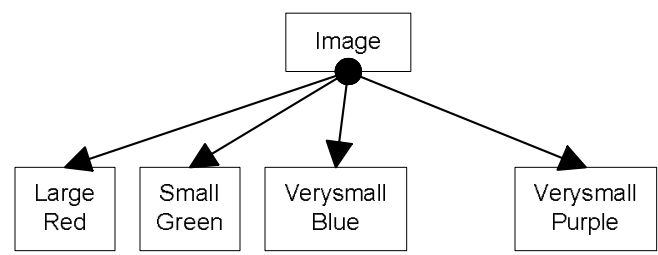

Figure 2. Fuzzy content representation for an image

Fuzzy terms for colour contents for each image: verysmall $->[0,0.3]$

small $->[0,0.4]$

rathersmall $->[0.25,0.45]$

medium $\rightarrow[0.30,0.70]$

ratherlarge $\rightarrow[0.55,0.75]$

large $->[0.60,1]$

verylarge- $>[0.70,1]$

\section{Image similarity}

Similarity measurement plays a vital role in contentbased image retrieval (CBIR), since without this concept of similarity measurement; the retrieval of images from a database would not be possible. After the process of feature extraction has been carried out on an image database, the stored image feature content must be compared in terms of similarity taking into account either colour, texture, or shape features. The use of this extracted feature data allows for a database to be indexed automatically according to its extracted features, rather than having manually index the entire database by keywords for example.

In this research, fuzzy logic based similarity is proposed between the two images. The weights are assigned to fuzzy colour content during the calculation of similarity between the two images.

Assigning the weights for each fuzzy content term: verysmall $\rightarrow 0.1$, small $\rightarrow 0.25$, rathersmall $\rightarrow 0.4$, medium $\rightarrow 0.55$, ratherlarge $\rightarrow 0.70$, large $\rightarrow 0.85$, verylarge- $>1$

A fuzzy intersection is the lower membership in both sets of each element. The fuzzy intersection of two fuzzy sets $A$ and $B$ on universe of discourse X:

$$
\mu A \cap B(x)=\min [\mu A(x), \mu B(x)]=\mu A(x) \cap \mu B(x),
$$

where $x \in X$

The union is the largest membership value of the element in either set. The fuzzy operation for forming the union of two fuzzy sets A and B on universe $\mathrm{X}$ can be given as:

$$
\mu \mathrm{A} \cup \mathrm{B}(\mathrm{x})=\max [\mu \mathrm{A}(\mathrm{x}), \mu \mathrm{B}(\mathrm{x})]=\mu \mathrm{A}(\mathrm{x}) \cup \mu \mathrm{B}(\mathrm{x}),
$$

where $\mathrm{x} \in \mathrm{X}$

Finally the fuzzy similarity distance is $\mid \mu \mathrm{A} \cup \mathrm{B}(\mathrm{x})$ $\mu A \cap B(x) \mid$

Based on the fuzzy image features and similarity measure, experiments were conducted on image database. Next section describes the experimental results.

\section{Experimental results}

To test the effectiveness of this proposed system, the preliminary experiments were conducted on a small colour image database. Around 1000 real world images were downloaded from various websites. This image database contains a wide variety of images, like the images of flowers, scenery, animals, mountains etc. Most of the images are in jpg format. All the nine colours were extracted from each image; those colours were converted into fuzzy terms before storing them in database.

To check the performance of the developed fuzzy based similarity measure, colour features were extracted and fuzzy contents were stored in database for each image. Some of the experimental results are discussed in this paper. Figure 3 shows the top eleven results returned for a query. The image- 1 represents a query image along with the retrieved images along with their fuzzy distance.

\section{Analysis and comparison}

In order to compare the performance of fuzzy image features and learning similarity measure based on fuzzy logic, various distance formulae were implemented and tested. The results are analysed using precision and recall method using the consistent criteria and image database for each similarity measure. Recall signifies the relevant images in the database that are retrieved in response to a query. Precision is the proportion of the retrieved images that are relevant to the query. More precisely, let $A$ be the set of relevant items, let $B$ the set of retrieved items and $a, b, c$ and $d$ are given in Figure 4. In the picture, $a$ stands for 'retrieved relevant' images, $b$ for 'retrieved irrelevant' images, $c$ for 'unretrieved relevant' images and $d$ for 'unretrieved irrelevant' images. Then recall and precision are defined as the following conditional probabilities [13]. 


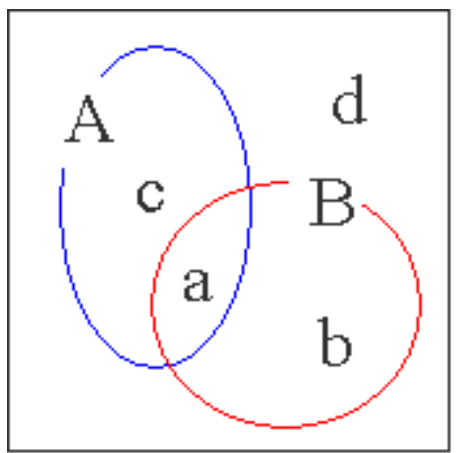

Figure 4. Sets for explaining retrieval effectiveness

$$
\begin{gathered}
\text { recall }=P(B \mid A)=\frac{P(A \cap B)}{P(A)}=\frac{a}{a+c}, \\
\text { precision }=P(A \mid B)=\frac{P(A \cap B)}{P(B)}=\frac{a}{a+b},
\end{gathered}
$$

For 1000 images, $82 \%$ of the recall and $87 \%$ precision has been achieved. With these conditions, image retrieval is said to be more effective if precision values are higher at the same recall values. Figure 5 shows the retrieval performance based on other similarity measures. Every similarity functions perform well except the histogram intersection. Euclidean distance has performed better than proposed fuzzy based similarity measure. The histogram intersection distance filters out the irrelevant elements in the matching of two feature vectors. Euclidean distance has performed better than fuzzy distance and has been used in many image retrieval systems to compare the two images based on their features. City block distance and chi-square are also desirable measures in terms of both retrieval effectiveness and efficiency.

\section{Conclusion}

This paper presents a problem of semantic gap, low level features extracted from image and high level query expressed by the user. It is very important to reduce this semantic gap to achieve the better retrieval results. The paper proposes a novel approach of fuzzy mapping on image database; therefore instead of storing the actual numerical values in database, fuzzy terms are stored for each colour for the image. While calculating the similarity based on query image, these terms are converted into numeric weights. Fuzzy based similarity function is used to calculate the similarity between the two images. Experiments were conducted on small image database and promising results were obtained.

\section{References}

[1] M. Flickner, H. Sawhney, W. Niblack, J. Ashley, Q. Huang, B. Dom, M. Gorkani, J. Hafner, D. Lee, D. Petkovic, D. Steele and P. Yanker, Query By Image and Video Content: The QBIC System, IEEE Computer, Vol. 28, Number 9, pp. 23-32, September 1995.

[2] A. Gupta, Visual Information Retrieval: A Virage Perspective, Technical Report Revision 4, Virage Inc, San Diego, CA 92121, 1996, URL: http://www.virage.com/wpaper.

[3] A. Pentland, R. Picard and S. Sclaroff, Photobook: Content-based Manipulation of Image Databases, International Journal of Computer Vision, Vol. 3, pp. 233-254, 1996.

[4] W. Ma and B. Manjunath, NETRA: A Toolbox for Navigating Large Image Databases, Journal of ACM Multimedia Systems, Vol. 7, Number 3, pp.184-198, 1999.

[5] J. Smith and S. Chang, Querying by Colour Regions Using the VisualSEEK Content-Based Visual Query System, Chapter in Intelligent Multimedia Information Retrieval, 1996, URL: http://www.ctr.columbia.edu/papers_advent/96/smith96 d.html.

[6] C. Carson, S. Belongie, H. Greenspan and J. Malik, Blobworld: Image Segmentation using ExpectationMaximization and Its Application to Image Querying, Journal of Pattern Analysis and Machine Intelligence, 1998, URL: http:// elib.cs.berkeley.edu/carson/papers/pami.html.

[7] V. Ogle and M. Stonebraker, Chabot: Retrieval from a Relational Database of Images, IEEE Computer, Volume 28, Number 9, pp. 40-48, 1995.

[8] B. Verma and S. Kulkarni, Fuzzy Logic Based Interpretation and Fusion of Colour Queries, Journal of Fuzzy Sets and Systems, Volume 147, Number 1, pp. 99-118, 2004.

[9] J. Han and K. Ma, Fuzzy Colour Histogram and its Use in Colour Image Retrieval, IEEE Transactions on Image Processing, Volume 11, Number 8, pp. 944-952, 2002.

[10] N. Sugano, Colour Naming System using Fuzzy Set Theoretical Approach, Proceedings of $10^{\text {th }}$ IEEE International Conference on Fuzzy Systems, Volume 1, pp. 81-84, 2001.

[11] A. Younes, I. Truck. H. Akdag, Image Retrieval using Fuzzy Representation of Colours, Journal of Soft Computing, Volume 11, pp. 287-298, 2007.

[12] J. Foley, A. Dam, S. Feiner and J. Hughes, Computer Graphics Principles and Practice, Addison-Wesley Publishing Company, 1990.

[13] J. Smith and S. Chang, Tools and Techniques for Colour Image Retrieval", In Symposium on Electronic Imaging: Science and Technology - Storage \& Retrieval for Image and Video Databases IV, Vol. 2670, San Jose, CA, February 1996. 


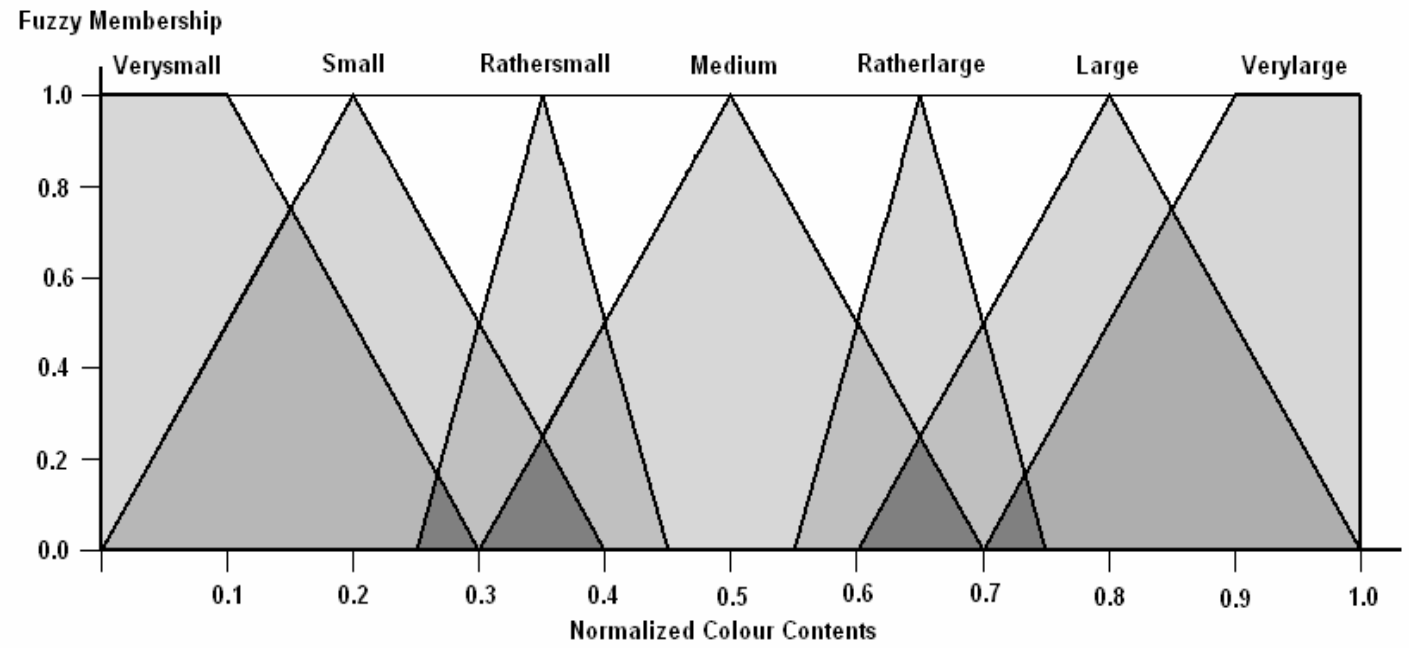

Figure 1. Fuzzy membership function for normalized colour contents

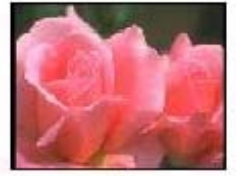

(1)

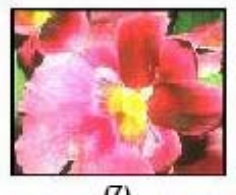

(7)

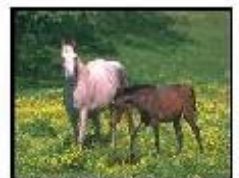

(1)

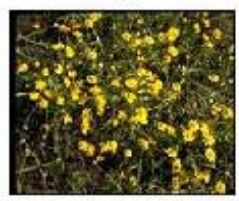

(7)

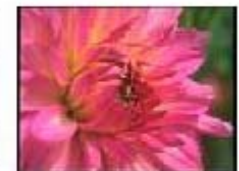

(2)

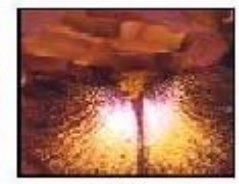

(8)

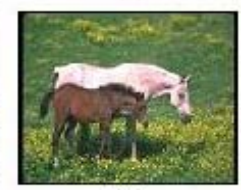

(2)

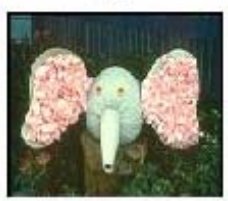

(8)

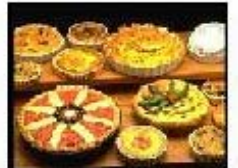

(3)

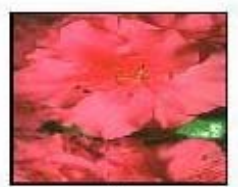

(9)

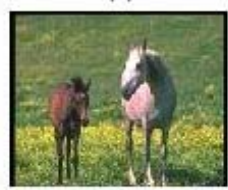

(3)

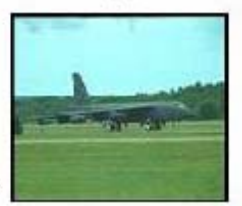

(9)

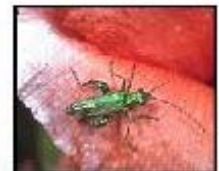

(4)

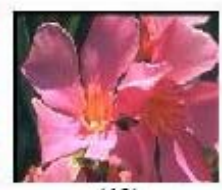

(10)

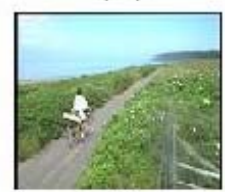

(4)

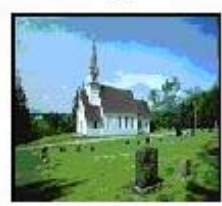

(10)

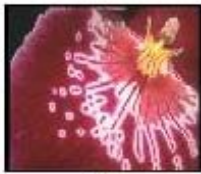

(5)

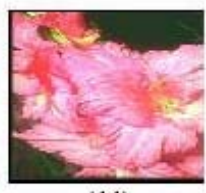

(11)

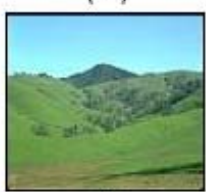

(5)

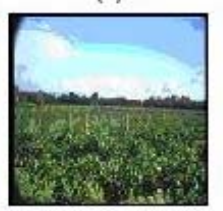

(11)

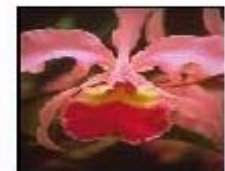

(6)

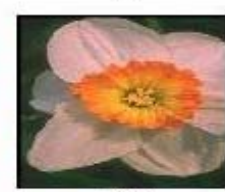

(12)

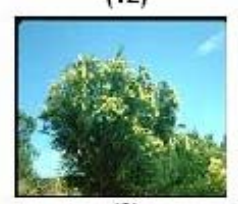

(6)

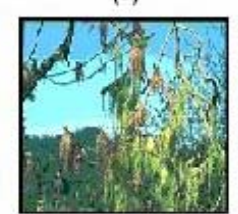

(12)

Figure 3. Query image (image-1) and retrieved images (image-2 to image-12) 


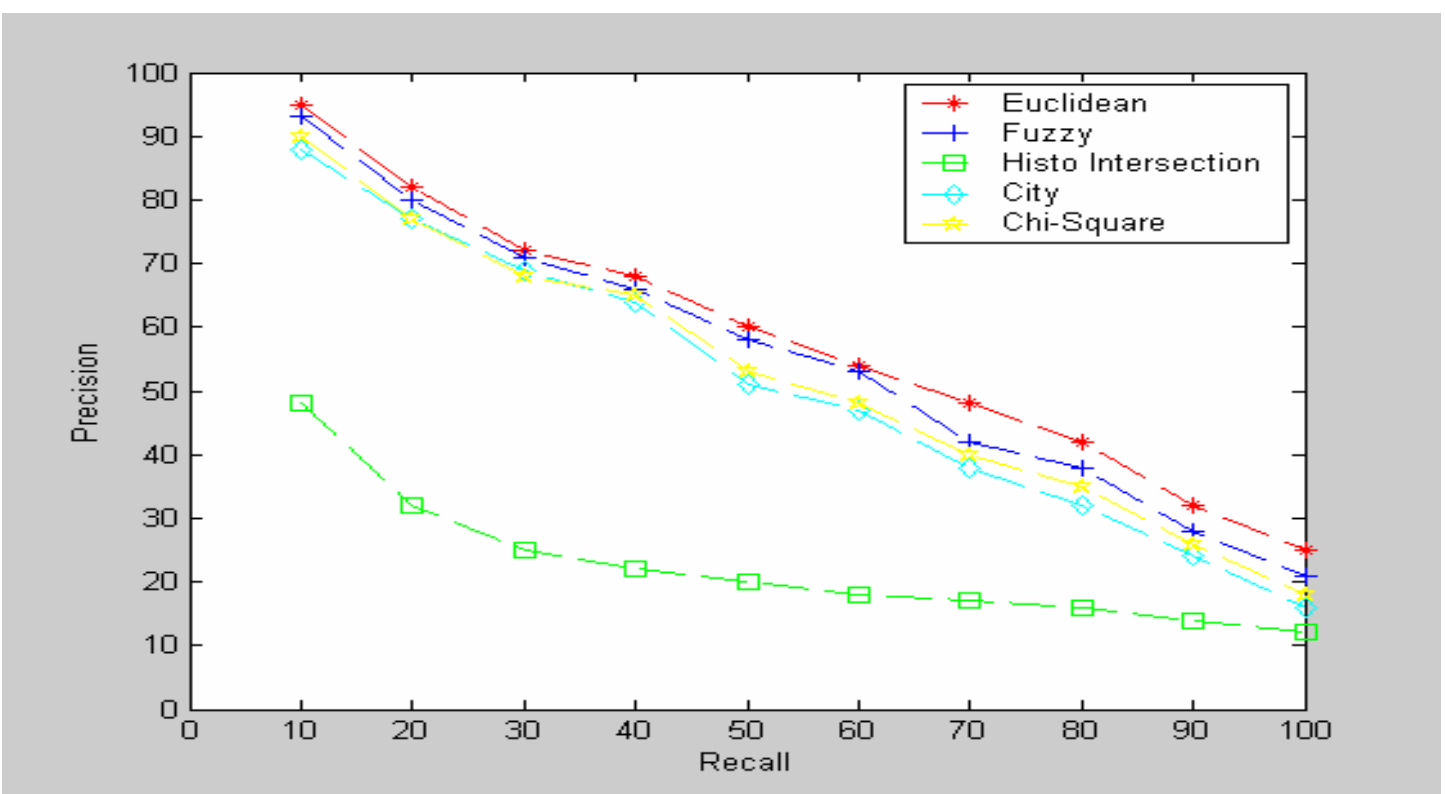

Figure 5. Comparison of retrieval performance for various similarity measures 